\title{
Association between antidepressant use and reduced risk of intubation or death in hospitalized patients with COVID-19: results from an observational study
}

\author{
Nicolas Hoertel $\mathbb{1}^{1,2,3} \cdot$ Marina Sánchez-Rico $\mathbb{1}^{1,4} \cdot$ Raphaël Vernet $\mathbb{C}^{5} \cdot$ Nathanaël Beeker ${ }^{6}$. \\ Anne-Sophie Jannot ${ }^{3,5,7} \cdot$ Antoine Neuraz $^{7,8} \cdot$ Elisa Salamanca ${ }^{9}$ Nicolas Paris ${ }^{10,11} \cdot$ Christel Daniel $^{10,12}$. \\ Alexandre Gramfort ${ }^{13}$. Guillaume Lemaitre ${ }^{13}$ - Mélodie Bernaux ${ }^{14}$ - Ali Bellamine ${ }^{6}$. Cédric Lemogne $\mathbb{1}^{1,2,3}$. \\ Guillaume Airagnes $^{1,2,3} \cdot$ Anita Burgun $^{7} \cdot$ Frédéric Limosin $^{1,2,3} \cdot$ On behalf of AP-HP / Universities / INSERM COVID- \\ 19 Research Collaboration and AP-HP COVID CDR Initiative
}

Received: 19 September 2020 / Revised: 18 December 2020 / Accepted: 7 January 2021 / Published online: 4 February 2021

(c) The Author(s), under exclusive licence to Springer Nature Limited 2021

\begin{abstract}
A prior meta-analysis showed that antidepressant use in major depressive disorder was associated with reduced plasma levels of several pro-inflammatory mediators, which have been associated with severe COVID-19. Recent studies also suggest that several antidepressants may inhibit acid sphingomyelinase activity, which may prevent the infection of epithelial cells with SARS-CoV-2, and that the SSRI fluoxetine may exert in-vitro antiviral effects on SARS-CoV-2. We examined the potential usefulness of antidepressant use in patients hospitalized for COVID-19 in an observational multicenter retrospective cohort study conducted at AP-HP Greater Paris University hospitals. Of 7230 adults hospitalized for COVID-19, 345 patients (4.8\%) received an antidepressant within $48 \mathrm{~h}$ of hospital admission. The primary endpoint was a composite of intubation or death. We compared this endpoint between patients who received antidepressants and those who did not in time-to-event analyses adjusted for patient characteristics, clinical and biological markers of disease severity, and other psychotropic medications. The primary analysis was a multivariable Cox model with inverse probability weighting. This analysis showed a significant association between antidepressant use and reduced risk of intubation or death (HR, 0.56; 95\% CI, 0.43-0.73, $p<0.001)$. This association remained significant in multiple sensitivity analyses. Exploratory analyses suggest that this association was also significant for SSRI and non-SSRI antidepressants, and for fluoxetine, paroxetine, escitalopram, venlafaxine, and mirtazapine (all $p<0.05$ ). These results suggest that antidepressant use could be associated with lower risk of death or intubation in patients hospitalized for COVID-19. Double-blind controlled randomized clinical trials of antidepressant medications for COVID-19 are needed.
\end{abstract}

\section{Introduction}

Global spread of the novel coronavirus SARS-CoV-2, the causative agent of coronavirus disease 2019 (COVID-19), has created an unprecedented infectious disease crisis worldwide [1, 2]. In the current absence of a vaccine or curative treatment, the search for an effective treatment for

Supplementary information The online version contains supplementary material available at https://doi.org/10.1038/s41380021-01021-4.

Nicolas Hoertel

nico.hoertel@yahoo.fr

Extended author information available on the last page of the article patients with COVID-19 among all available medications is urgently needed [3, 4].

Both COVID-19 and severe acute respiratory syndrome (SARS) are characterized by an overexuberant inflammatory response [5] and, for COVID-19, viral load is associated with the worsening of symptoms [6].

Accumulating evidence suggests that severe COVID-19 is associated with an increased plasma level of inflammatory mediators including cytokines and chemokines such as interleukin (IL)-2, IL-6, IL-7, IL-10, tumor necrosis factor alpha (TNF- $\alpha$ ), monocyte chemoattractant protein-1 (MCP1; also known as CCL2), macrophage inflammatory protein 1 alpha (MIP1 $\alpha$; also known as CCL3), C-reactive protein, ferritin, and D-dimers [7]. A recent meta-analysis [8] of studies conducted in individuals with major depressive disorder following antidepressant treatment, mostly including selective serotonin 
reuptake inhibitors (SSRIs), supports that, overall, antidepressants may be associated with decreased plasma levels of 4 of 16 tested inflammatory mediators, including IL-10, TNF$\alpha$, and CCL-2, which are associated with COVID-19 severity [7], as well as IL-6, which is highly correlated with disease mortality $[7,9]$.

Furthermore, a recent study [10] suggests that functional inhibitors of acid sphingomyelinase activity (FIASMA), including several SSRI and non-SSRI antidepressants, may prevent the infection of epithelial cells with SARS-CoV-2 [11].

A recent in-vitro study [12] also supports antiviral effects of the SSRI fluoxetine on SARS-CoV-2, although this effect was not observed for other SSRIs, including paroxetine and escitalopram.

Finally, a recent randomized trial suggests that fluvoxamine, a SSRI, sigma-1 receptor (S1R) agonist and FIASMA, may prevent clinical deterioration in outpatients with acute COVID-19 compared to placebo [13].

In this context, we hypothesized that antidepressants could be potentially useful in reducing the risk of intubation or death in patients with COVID-19. Short-term use of low to moderate doses of antidepressants, and particularly of SSRIs, is generally well tolerated [14], and notably in older adults [15-17].

To our knowledge, no clinical study has examined to date the potential usefulness of antidepressants in patients hospitalized for COVID-19. Observational studies of patients with COVID-19 taking medications for other indications can help decide which treatment should be prioritized for randomized clinical trials and minimize the risk for patients of being exposed to potentially harmful and ineffective treatments.

We took advantage of the Assistance Publique-Hôpitaux de Paris (AP-HP) Health Data Warehouse, which includes data on all patients with COVID-19 who had been consecutively admitted to any of the 39 AP-HP Greater Paris University hospitals.

In this report, we examined the association between antidepressant use and the risk of intubation or death among adult patients who have been admitted to these medical centers with COVID-19. If a significant protective association were found, we sought to perform exploratory analyses to examine whether this association may be specific to certain classes of antidepressants (i.e. SSRIs, serotonin-norepinephrine reuptake inhibitors (SNRIs), tricyclic, tetracyclic and $\alpha 2$-antagonist antidepressants) or certain individual antidepressant medications, in order to help guide future studies. Our main hypothesis was that antidepressant use would be associated with reduced risk of intubation or death in time-to-event analyses adjusting for patient characteristics, clinical and biological markers of disease severity, and other psychotropic medications.

\section{Methods}

\section{Setting}

We conducted a multicenter observational retrospective study at AP-HP, which includes 39 hospitals of which 23 are acute, 20 are adult and 3 are pediatric hospitals. We included all adults aged 18 years or over who have been admitted with COVID-19 to these medical centers from the beginning of the epidemic in France, i.e. January 24th, until April 1st. COVID-19 was ascertained by a positive reverse-transcriptase-polymerase-chain-reaction (RTPCR) test from analysis of nasopharyngeal or oropharyngeal swab specimens. This observational study using routinely collected data received approval from the Institutional Review Board of the AP-HP clinical data warehouse (decision CSE-20-20_COVID19, IRB00011591, April 8th, 2020). AP-HP clinical Data Warehouse initiative ensures patient information and informed consent regarding the different approved studies through a transparency portal in accordance with European Regulation on data protection and authorization $\mathrm{n}^{\circ} 1980120$ from National Commission for Information Technology and Civil Liberties (CNIL). All procedures related to this work adhered to the ethical standards of the relevant national and institutional committees on human experimentation and with the Helsinki Declaration of 1975, as revised in 2008.

\section{Data sources}

We used data from the AP-HP Health Data Warehouse ('Entrepôt de Données de Santé (EDS)'). This warehouse contains all the clinical data available on all inpatient visits for COVID-19 to any of the 39 AP-HP Greater Paris University hospitals. The data obtained included patient demographic characteristics, vital signs, laboratory test and RT-PCR test results, medication administration data, medication lists during current and past hospitalizations in AP-HP hospitals, current diagnoses, discharge disposition, ventilator use data, and death certificates.

\section{Variables assessed}

We obtained the following data for each patient at the time of the hospitalization through electronic health records: [17-19] sex, age, hospital, obesity, self-reported current smoking status, any medical condition associated with increased risk of severe COVID-19 [17, 19-21], any medication prescribed according to compassionate use or as part of a clinical trial [22], clinical and biological markers of disease severity at admission [23, 24], any current mood or 
anxiety disorder, any other current psychiatric disorder, and any psychotropic medication, including any antidepressant, benzodiazepine or Z-drug, mood stabilizer (i.e. lithium or antiepileptic medications with mood stabilizing effects), and antipsychotic medication [25, 26]. These variables are detailed in Supplementary Text 1.

\section{Antidepressant use}

Study baseline was defined as the date of hospital admission. Antidepressant use was defined as receiving any antidepressant within the first $48 \mathrm{~h}$ of hospital admission and before the end of the index hospitalization or intubation or death. We used this delay because we considered that, in a context of overwhelming of all hospital units during the COVID-19 peak incidence, patients may not have received or been prescribed the treatment the first day of their admission.

\section{Primary endpoint}

The primary endpoint was the time from study baseline to intubation or death. For patients who died after intubation, the timing of the primary endpoint was defined as the time of intubation. Patients without an end-point event had their data censored on May 20th, 2020.

\section{Statistical analysis}

We calculated frequencies and means ( \pm standard deviations (SD)) of each baseline characteristic described above in patients receiving or not receiving antidepressants and compared them using chi-square tests or Welch's $t$-tests.

To examine the associations between the use of any antidepressant, each class of antidepressants, and each individual antidepressant with the composite endpoint of intubation or death, we performed Cox proportional-hazards regression models. To help account for the nonrandomized prescription of antidepressants and reduce the effects of confounding, the primary analyses used a propensity score analysis with inverse probability weighting $[27,28]$. The individual propensities for exposures were estimated by multivariable logistic regression models that included sex, age, hospital, obesity, smoking status, any medical condition, any medication prescribed according to compassionate use or as part of a clinical trial, clinical and biological markers of severity of COVID-19, the presence of mood or anxiety or other current psychiatric disorders, and any prescribed benzodiazepine or Z-drug, mood stabilizer, and antipsychotic medication. In the inverse-probability-weighted analyses, the predicted probabilities from the propensity-score models were used to calculate the stabilized inverse-probability-weighting weights [27]. Associations of any antidepressant, each class of antidepressants, and each individual antidepressant with the primary endpoint were then estimated using multivariable Cox regression models including the inverse-probabilityweighting weights. Kaplan-Meier curves were performed using the inverse-probability-weighting weights [29], and their pointwise $95 \%$ confidence intervals were estimated using the nonparametric bootstrap method [30].

We conducted sensitivity analyses, including multivariable Cox regression models comprising as covariates the same variables as the inverse-probability-weighted analyses, and univariate Cox regression models in matched analytic samples. For these latter analyses, we decided a priori to select one control for each exposed case for exposures to any antidepressant and each class of antidepressants, and two controls for each exposed case for individual antidepressant medications, based on the same variables used for both the inverse-probability-weighted and the multivariable Cox regression analyses. Weighted Cox regression models were used when proportional hazards assumption was not met. To reduce the effects of confounding, optimal matching was used in order to obtain the smallest average absolute distance across all clinical characteristics between exposed patients and non-exposed matched controls.

We also performed several additional analyses. First, to increase our confidence that the results might not be due to unmeasured confounding or indication bias, we examined whether the primary endpoint differed between patients receiving an antidepressant only in the 3 months before hospital admission (during a prior hospitalization in a APHP hospital) and those who received it during the visit only. Second, to examine a potential immortal bias in the exposed group, we performed additional Cox regression analyses to compare the potential effect of antidepressant use to that of an active comparator, i.e., the benzodiazepine diazepam. Third, we performed multivariable Cox regression models including interaction terms to examine whether the association between antidepressant use and the endpoint significantly differed across subgroups defined by baseline characteristics. Fourth, we examined a potential dose-effect relationship by testing the association between the daily dose received (converted into fluoxetine-equivalent dose [31] and dichotomized at the median value) with the endpoint among patients receiving antidepressants. Fifth, we examined whether exposure to a combination of antidepressants was associated with a significantly different risk of intubation or death than exposure to only one antidepressant. Finally, we reproduced the main analyses (i) among patients with critical COVID-19 hospitalized in intensive care units (ICUs) and (ii) using death and intubation as separate endpoints.

For all associations, we performed residual analyses to assess the fit of the data, check assumptions, including proportional hazards assumptions, and examined the 
potential influence of outliers. We followed the recommendations of The Strengthening the Reporting of Observational Studies in Epidemiology (STROBE) Initiative [32]. Our main analysis focused on the association between antidepressant use and the primary endpoint. Statistical significance was fixed a priori at two-sided $p$ value $<0.05$. Only if a significant protective association were found, we planned to perform exploratory analyses and examine the associations between each class of antidepressants and each individual antidepressant medication with the endpoint. All analyses were conducted in R software version 2.4.3 (R Project for Statistical Computing).

\section{Results}

\section{Characteristics of the cohort}

Of the 9509 patients consecutively admitted to 33 participating AP-HP Greater Paris University hospitals with
COVID-19, 2164 patients (22.8\%) were excluded because of missing data or their young age (i.e. less than 18 years of age). In addition, 115 patients who were prescribed an antidepressant were excluded because they received it more than $48 \mathrm{~h}$ from hospital admission or after intubation. Of the remaining 7230 adult inpatients, 345 patients $(4.8 \%)$ received an antidepressant within $48 \mathrm{~h}$ of hospital admission at a mean fluoxetine-equivalent dose of $21.6 \mathrm{mg}$ ( $\mathrm{SD}=$ 14.1) per day (Fig. 1). Doses of each antidepressant medication are described in Supplementary Table 1. Among patients exposed to antidepressants, 298 (86.4\%) were exposed to only one antidepressant, and $47(15.8 \%)$ were exposed to at least two antidepressants.

First positive COVID-19 RT-PCR tests were obtained after a median delay of 1 day $(\mathrm{SD}=12.0)$ from study baseline. This delay was not significantly different between patients receiving or not receiving antidepressants [median in the exposed group $=1$ day $(\mathrm{SD}=14.1)$; median in the non-exposed group $=1$ day $(\mathrm{SD}=11.9)$; Mood's median test Chi-square $=0.21, p=0.650)]$.
Fig. 1 Study cohort.

Antidepressant use was defined as receiving any antidepressant during the first $48 \mathrm{~h}$ of hospital admission and before the end of the index hospitalization or intubation or death. SSRIs selective serotonin reuptake inhibitors, SNRIs serotonin-norepinephrine reuptake inhibitors.

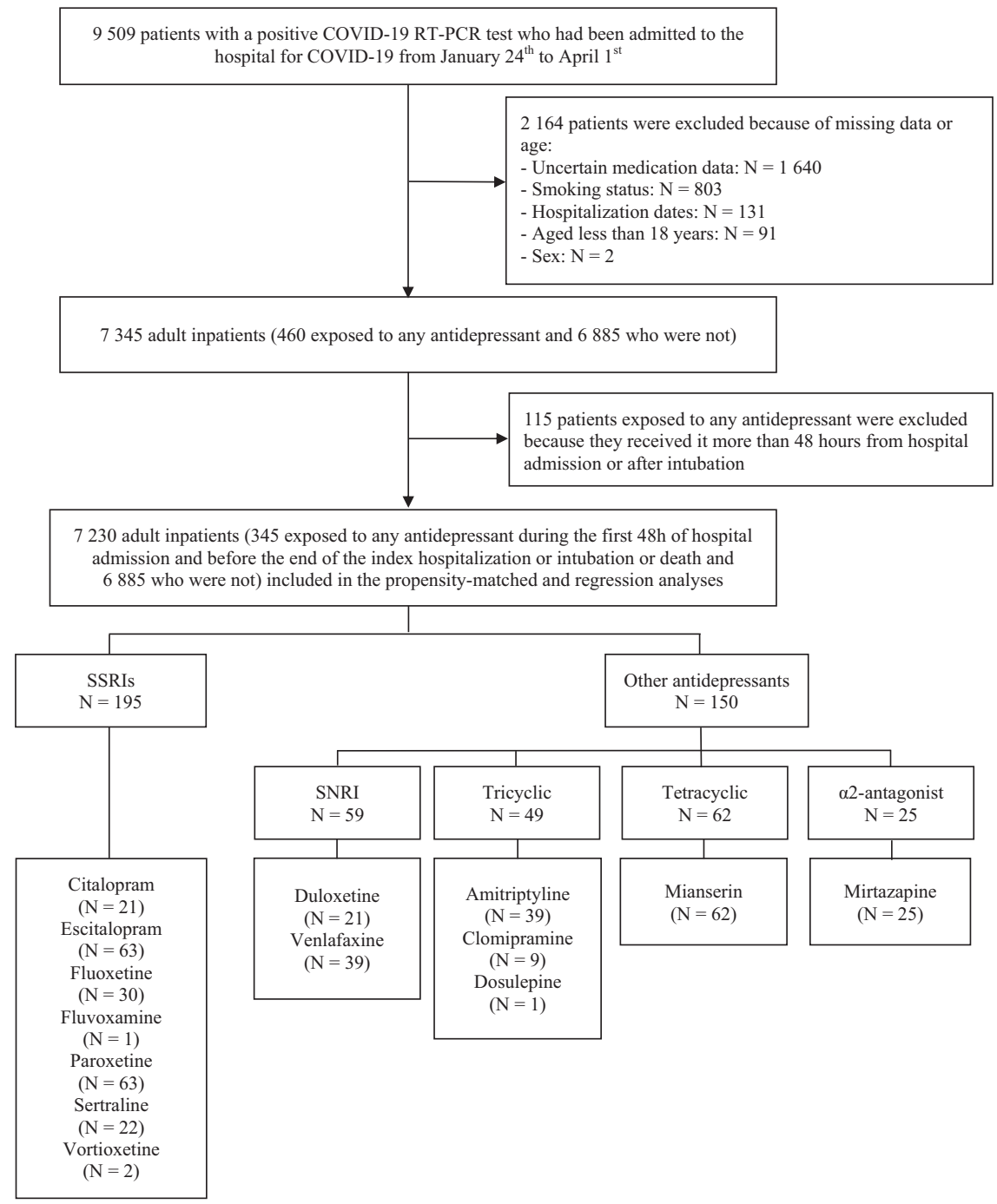


Over a mean follow-up of 18.4 days $(\mathrm{SD}=27.2$; median $=5$ days; range: $1-117$ days), 1272 patients $(18.1 \%)$ had a primary end-point event at the time of data cutoff on May 20th. Times to follow-up by antidepressant medication are shown in Supplementary Table 2.

All baseline characteristics, when examined independently, were significantly associated with the endpoint (Table 1). In the multivariable analysis, these associations remained significant, except for current smoking, any medication prescribed according to compassionate use or as part of a clinical trial, any current mood or anxiety disorder, any other current psychiatric disorder, any mood stabilizer medication, and any antipsychotic medication (Table 1).

The distributions of patient characteristics according to antidepressant use, and exposure by class of antidepressants and by individual medication are shown in Supplementary Tables 3-13. In the full sample, antidepressant use and exposure to each antidepressant class and each individual medication significantly differed according to most baseline characteristics. The direction of these associations indicated older age [mean age of patients exposed to antidepressants was $73.7(\mathrm{SD}=15.8$ ) years, whereas it was $56.8(\mathrm{SD}=$ 19.3) years in those who were not] and overall greater medical severity of patients who received antidepressants than those who did not. After applying the propensity score weights, these differences were substantially reduced (Supplementary Tables 3-13). In the matched analytic samples, there were no significant differences in patient characteristics across the different exposures, except for exposures to any antidepressant, non-SSRI antidepressants and SNRI antidepressants, for which sex ratio significantly differed (Supplementary Tables 3-13).

\section{Study endpoint}

Among patients receiving any antidepressant, SSRIs, and non-SSRI antidepressants, the primary endpoint of intubation or death occurred respectively in 84 patients $(24.3 \%)$, 45 patients $(23.1 \%)$ and 39 patients $(26.0 \%)$, while 1188 non-exposed patients $(17.3 \%)$ had this outcome (Table 2). Unadjusted hazard ratio age-stratified estimates of the association between antidepressant use and the endpoint were non-significant (all $p>0.060$ ) (Supplementary Table 14). Following adjustments, the primary multivariable analyses with inverse probability weighting showed significant associations between use of any antidepressant (HR, 0.56; 95\% CI, 0.43-0.73, $p<0.001$ ) (Fig. 2), SSRI antidepressants (HR, 0.51; 95\% CI, $0.316-0.72, p<0.001)$, and non-SSRI antidepressants (HR, $0.65 ; 95 \%$ CI, 0.45-0.93, $p=0.018$ ) (Fig. 3) with reduced risk of intubation or death (Table 2). Among non-SSRI antidepressants, there were no significant associations between the endpoint and exposures to each class of antidepressants, except for $\alpha 2$-antagonist antidepressants (HR, 0.22; 95\% CI, 0.05-0.93, $p=0.040$ ) (Table 2; Fig. 3). Finally, exposure to escitalopram, fluoxetine, paroxetine, venlafaxine or mirtazapine was significantly associated with reduced risk of intubation or death (all $p<0.05)$. There were no significant differences in this risk according to exposure to any other molecule (Table 2; Supplementary Figs. 1-5).

In sensitivity analyses, multivariable Cox regression models in the full sample yielded similar results, as did univariate Cox regression models in the matched analytic samples, except for the association between SNRI antidepressants and the endpoint, which was significant in both sensitivity analyses, and that between $\alpha 2$-antagonist antidepressants and the outcome, which was not significant in the matched analytic sample (Table 2; Supplementary Figs. 1-5).

Additional analyses indicated that the association between antidepressant use and the endpoint was significant in both genders (Supplementary Table 15) and in a subsample of patients excluding those receiving an antipsychotic medication, who might be at increased risk for immune dysregulation linked to a psychotic disorder (Supplementary Table 16), supporting the robustness of our results. This association did not significantly differ across subgroups defined by baseline characteristics (Supplementary Table 17). Patients who were prescribed an antidepressant in the three months before but not during the visit were significantly at higher risk of intubation or death than those who received an antidepressant only during the visit (Supplementary Table 18). When considering diazepam as an active comparator, we found that antidepressant use was significantly associated with reduced risk of intubation or death in the crude (HR, 0.50; 95\% CI, 0.32-0.77, $p=0.002)$ and in the main (HR, 0.47; 95\% CI, 0.25-0.88, $p=0.018$ ) analyses, as well as in sensitivity analyses (Supplementary Table 19). Participants receiving diazepam did not have a significantly different risk of intubation or death compared to those not receiving either medication. Exposure to higher rather than lower doses of antidepressants was not significantly associated with the endpoint, except for tetracyclic antidepressants, for which higher doses were associated with an increased risk (Supplementary Table 20). Exposure to a combination of antidepressants was not significantly associated with the endpoint compared to exposure to only one antidepressant (Supplementary Table 21). When using intubation and death as separate endpoints, we found that antidepressant use was significantly associated with lower risks of death and intubation (Supplementary Table 22). Finally, among the 577 patients admitted to ICUs, antidepressant use was significantly associated with reduced risk of intubation or death (Supplementary Table 23). A post-hoc analysis of statistical power is presented in Supplementary Text 2. 


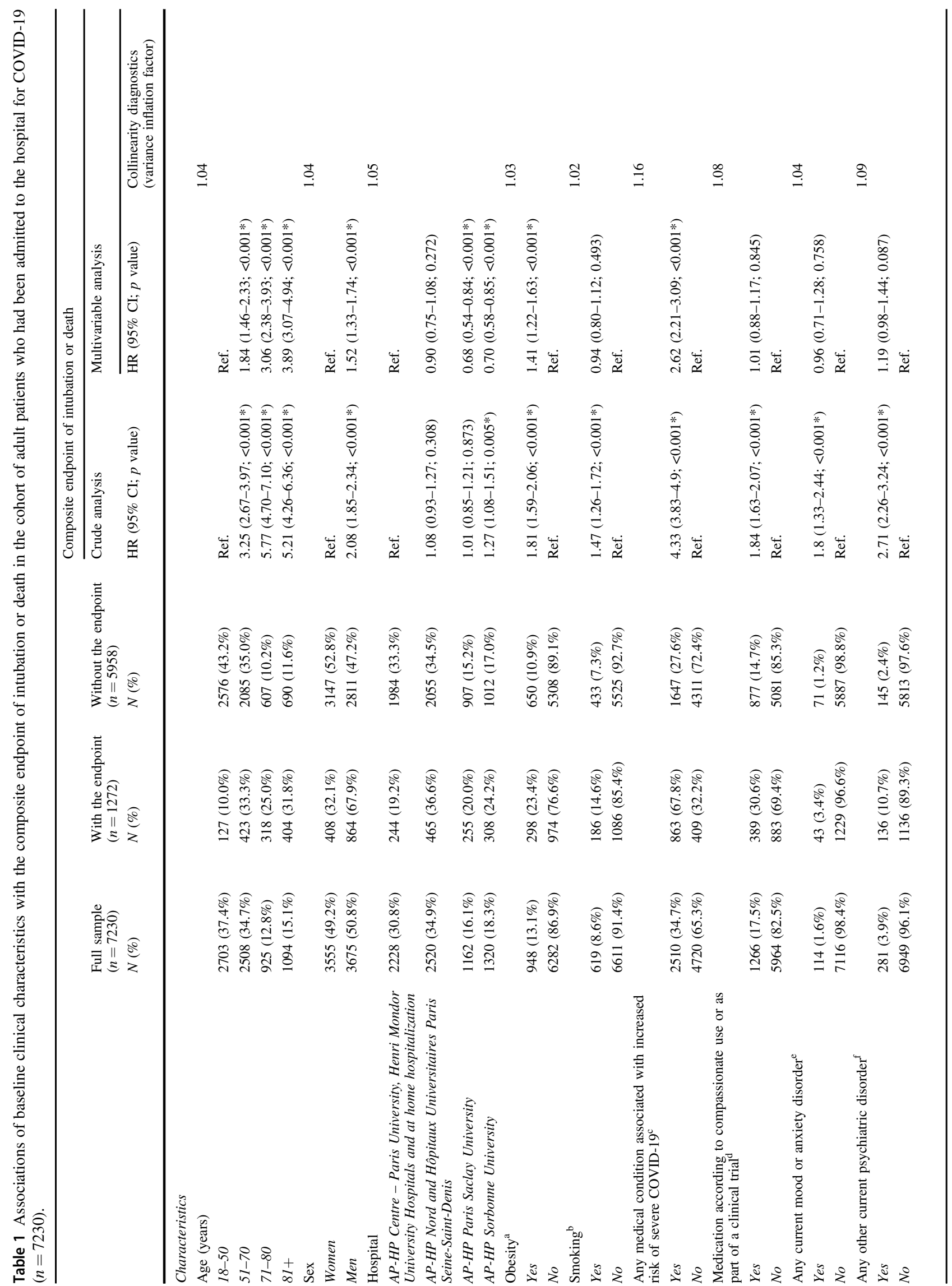




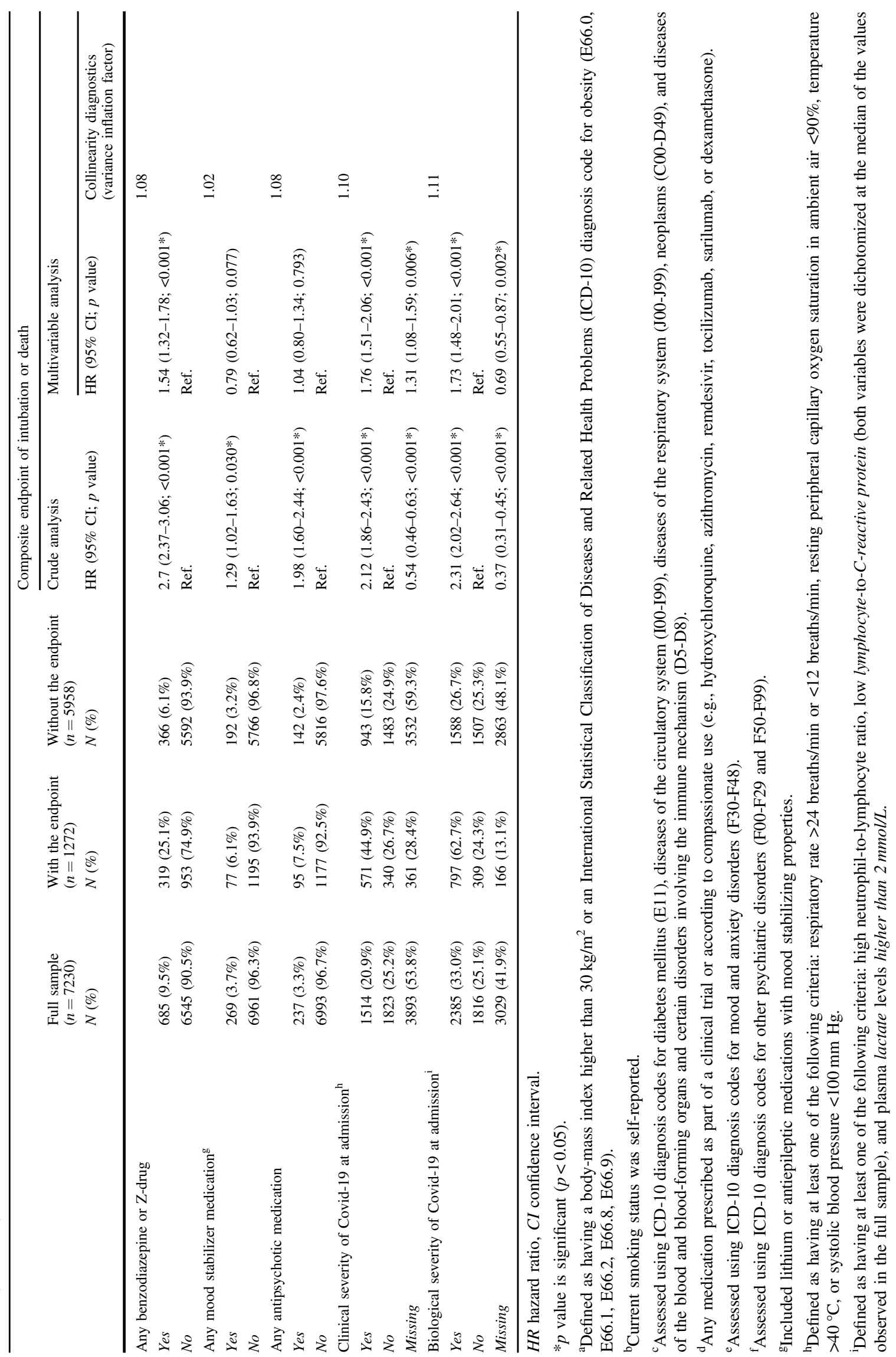




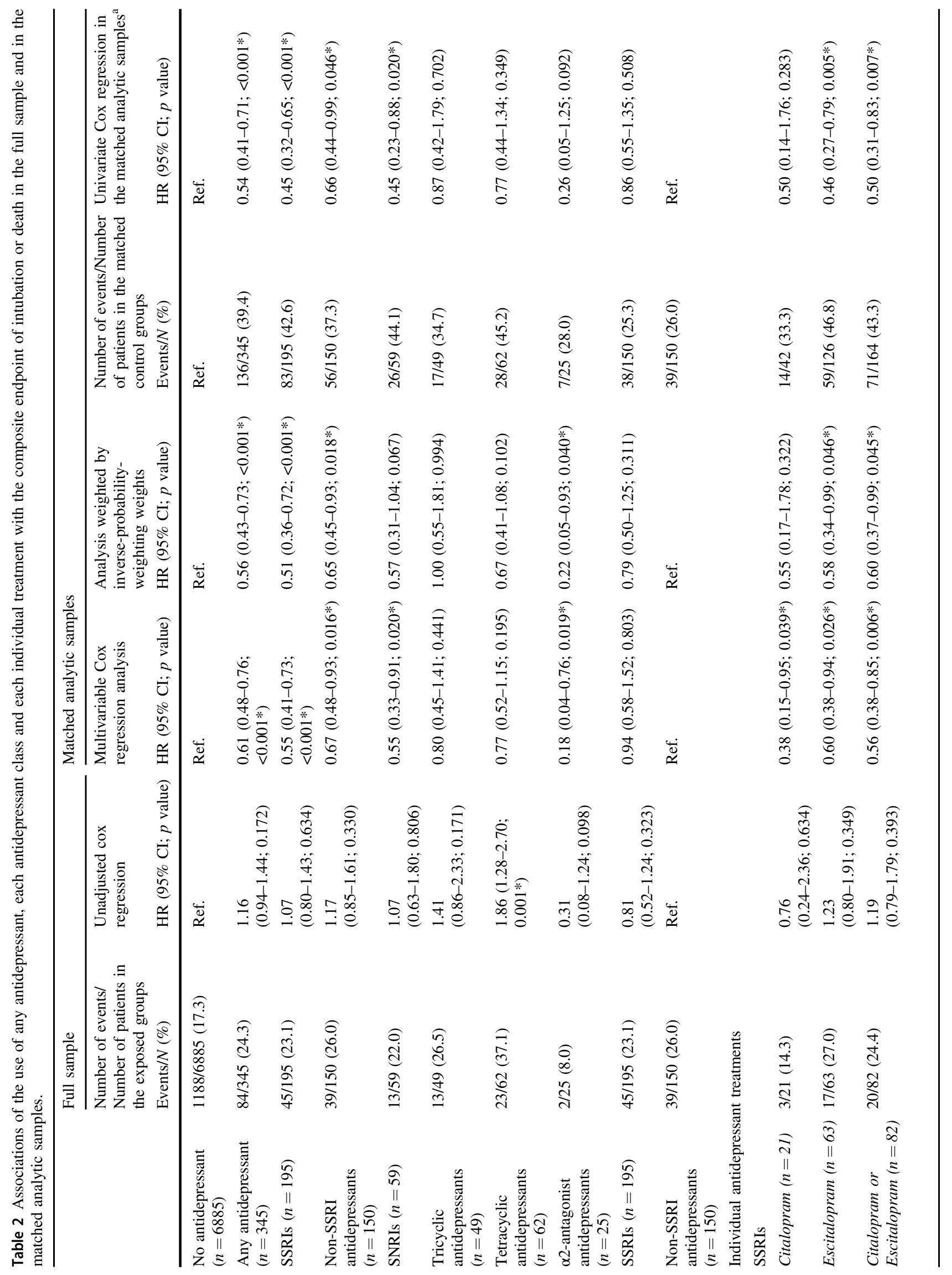




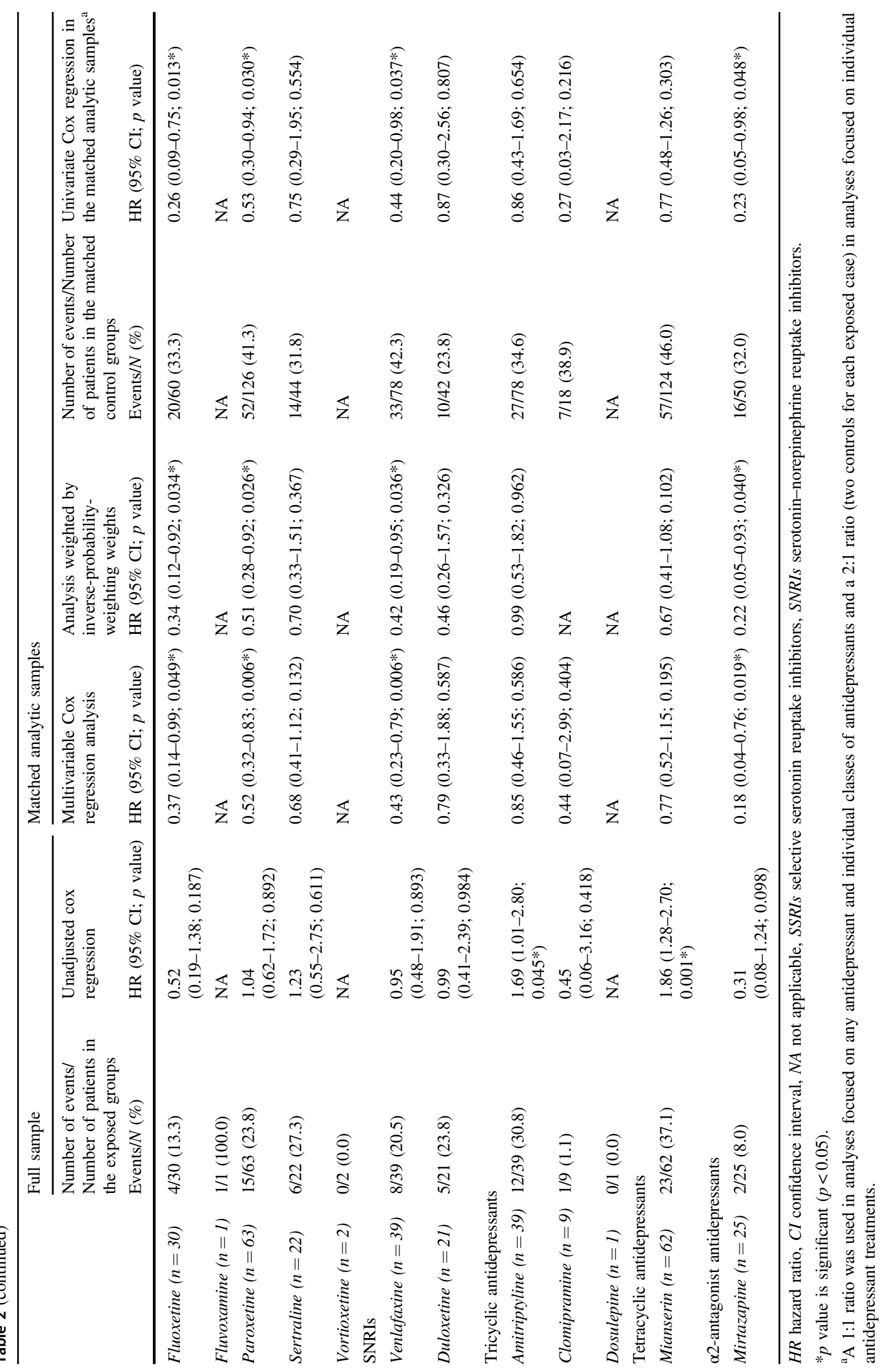



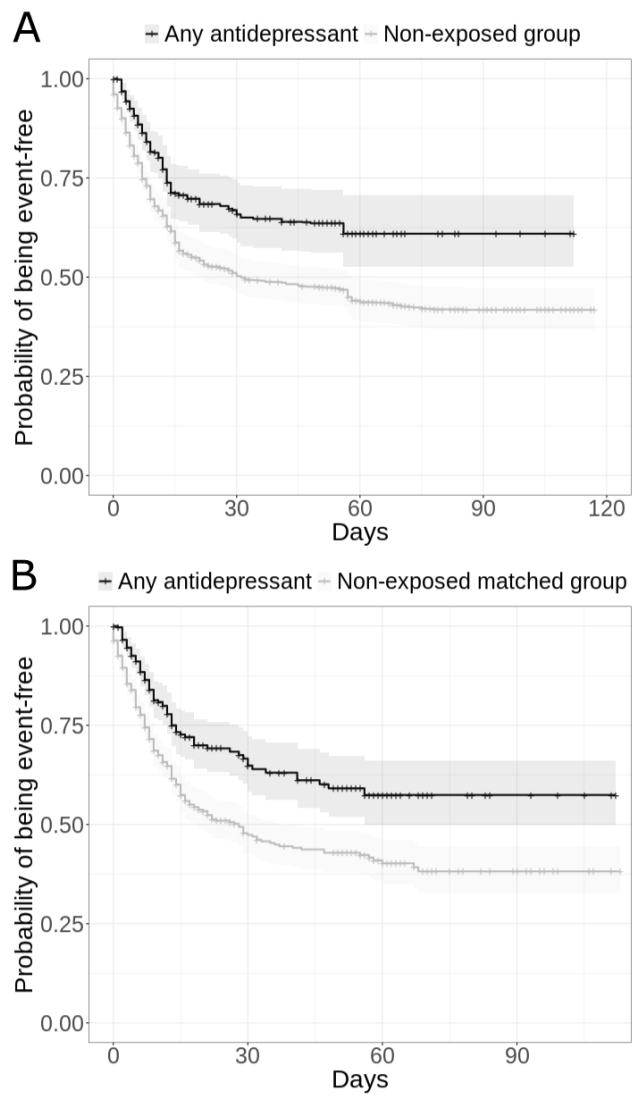

Fig. 2 Association between antidepressant use and the composite endpoint of intubation or death. Kaplan-Meier curves of the composite endpoint of intubation or death in the full sample $(n=7230)$ (A) and in the matched analytic sample using a 1:1 ratio $(n=648)(\mathbf{B})$ of patients who had been admitted with Covid-19, according to antidepressant exposure. The shaded areas represent pointwise $95 \%$ confidence intervals.

\section{Discussion}

In this multicenter retrospective observational study involving a large sample of patients hospitalized for COVID-19, we found that antidepressant use, at a mean dosage of 21.6 $(\mathrm{SD}=14.1)$ fluoxetine-equivalent milligrams, was significantly and substantially associated with reduced risk of intubation or death, independently of patient characteristics, clinical and biological markers of disease severity, and other psychotropic medications. This association remained significant in multiple sensitivity analyses. Exploratory analyses suggest that SSRI and non-SSRI antidepressants, and specifically the SSRIs escitalopram, fluoxetine, and paroxetine, and the SNRI venlafaxine, were significantly associated with reduced risk of intubation or death. These findings should be interpreted with caution due to the observational design of the study. However, our findings provide support for conducting controlled randomized clinical trials of antidepressant medications for COVID-19.
The association observed between antidepressant use and reduced risk of intubation or death might be explained by several mechanisms. First, prior studies suggest that several antidepressants may inhibit acid sphingomyelinase activity [10], which may prevent the infection of epithelial cells with SARS-CoV-2 [11]. Second, numerous antidepressants are S1R agonists [13], which might prevent the cytokine storm observed in severe COVID-19 [3]. Third, antidepressant use may be associated with reduced plasma levels of several inflammatory mediators associated with severe COVID-19 [7], including IL-6, IL-10, TNF- $\alpha$, and CCL-2 [8]. Finally, certain antidepressants may exert antiviral effects on SARS-CoV-2, such as fluoxetine [12]. If this link were confirmed, studies would be needed to determine the exact mechanisms underlying this association.

We found that individuals with psychiatric disorders and hospitalized for COVID-19 would be at higher risk of intubation or death, in line with findings of recent studies $[33,34]$. Our results suggest that this association may be explained by the greater prevalence of clinical characteristics associated with severe COVID-19 in individuals with psychiatric disorders than their counterparts, including medical comorbidity, and clinical and biological markers of disease severity. Nevertheless, future studies focusing specifically on this vulnerable population are needed to examine this issue.

Our study has several limitations. First, there are two possible major inherent biases in observational studies: unmeasured confounding and confounding by indication. We tried to minimize the effects of confounding in several different ways. First, we used multivariable regression models with inverse probability weighting to minimize the effects of confounding by indication [27, 28]. Second, we performed sensitivity analyses that showed similar results. Third, although some amount of unmeasured confounding may remain, our analyses adjusted for numerous potential confounders. Furthermore, our findings indicate that the association between antidepressant use and the endpoint was only observed for patients receiving an antidepressant during the visit and not in those who received it only within the 3 months before hospital admission. Fourth, this association remained significant when using death and intubation as separate endpoints. Fifth, the low number of patients with antidepressants admitted to ICUs in our study (12 of 577 patients, i.e. $2.1 \%$ ) and the significant association between antidepressant use and the endpoint in these patients further give strength to our conclusion. Finally, when considering diazepam as an active comparator, we found similar results.

Other limitations include missing data for some baseline characteristic variables, including baseline clinical and biological markers of severity of COVID-19, which may be explained by the overwhelming of all hospital units during 
Fig. 3 Associations of SSRIs and other antidepressants with the composite endpoint of intubation or death.

Kaplan-Meier curves of the composite endpoint of intubation or death in the full sample (A, $\mathbf{C}$ and $\mathbf{E}$ ) and in the matched analytic samples using a $1: 1$ ratio $(\mathbf{B}, \mathbf{D}$ and $\mathbf{F})$ of patients who had been admitted with Covid-19, according to exposure to antidepressant classes. The shaded areas represent pointwise $95 \%$ confidence intervals.
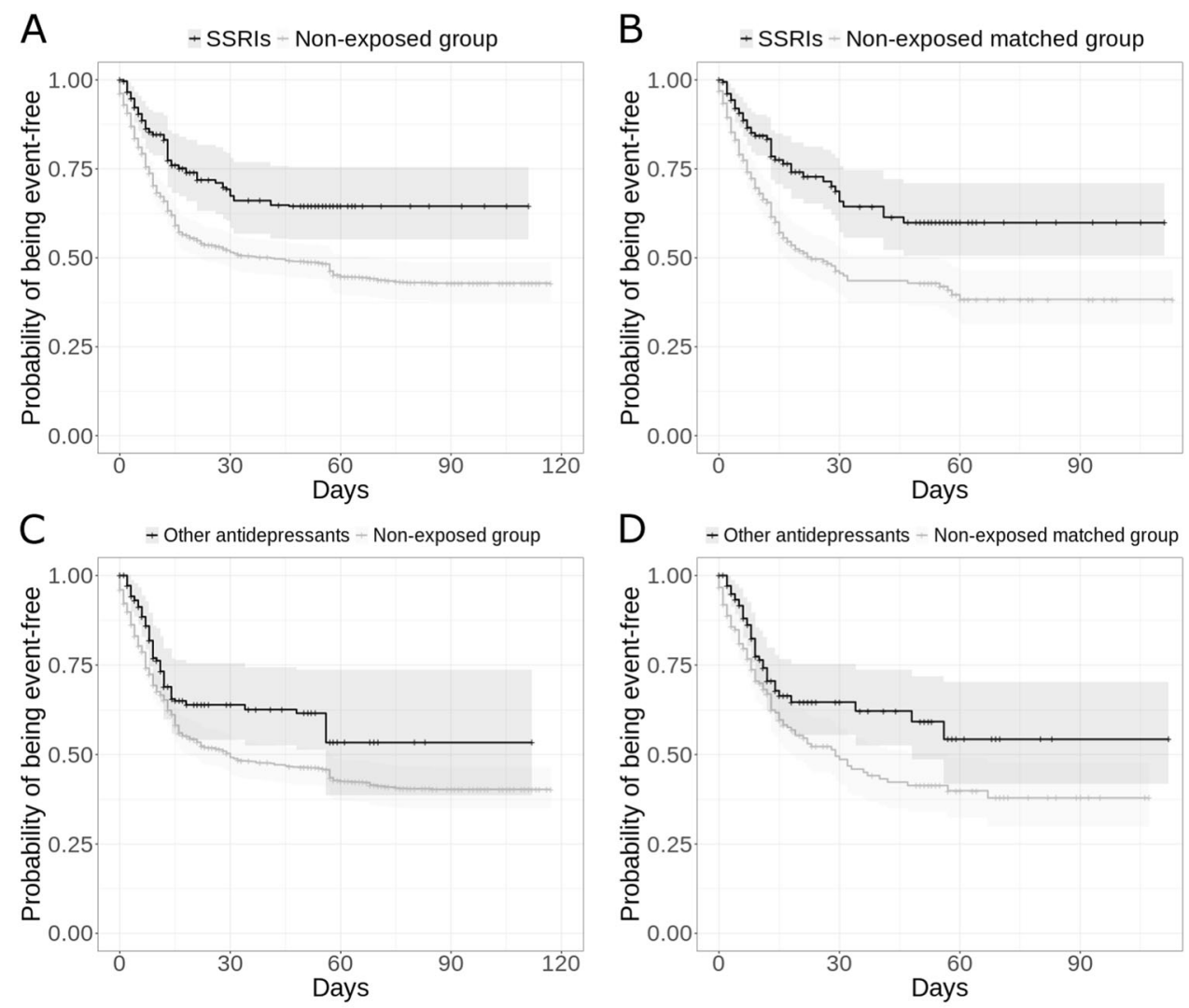

D + Other antidepressants - Non-exposed matched group
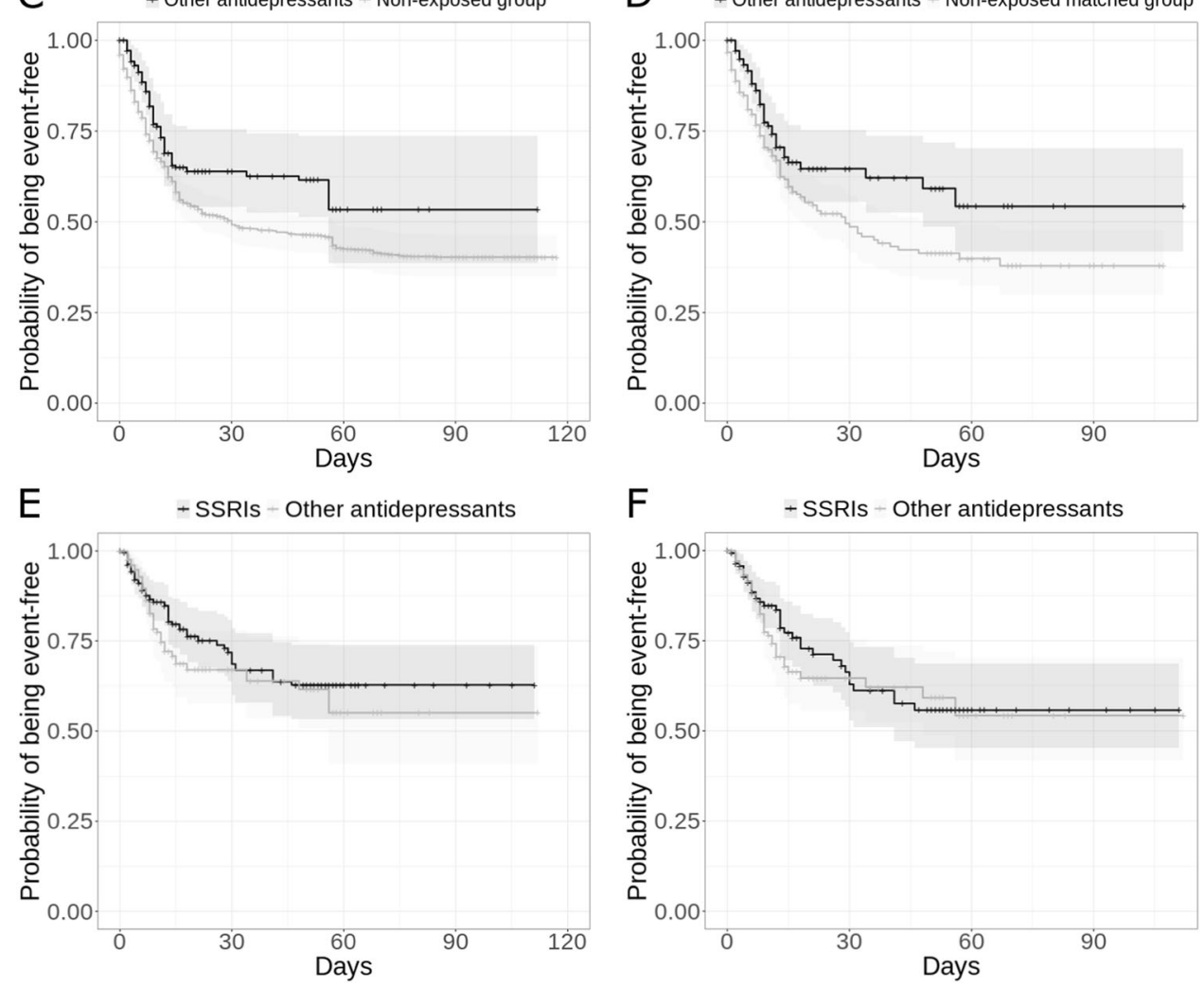

the COVID-19 peak incidence, and potential for inaccuracies in electronic health records in this context, especially for hand-written medical prescriptions. However, most associations observed between baseline characteristics and mortality or intubation are in line with prior epidemiological studies [17, 19]. Second, our study cannot establish a causal relationship between antidepressant use and reduced risk of intubation or death [35]. Third, inflation of type I error might have occurred in exploratory analyses due to multiple testing. Fourth, information on the reason for prescribing the antidepressant, the duration and adherence to its prescription, the prescription record of patients before the admission, and the date that COVID-19 symptoms appeared, was not available. Fifth, the criteria used to determine whether a patient with COVID-19 should be hospitalized or not might have varied during the study period. However, its length was relatively limited (i.e., from January 24th until April 1st) and our main results remained unchanged following additional adjustment for it (Supplementary Tables 24-26). Finally, despite the multicenter design, our results may not be generalizable to outpatients and other countries.

In this multicenter observational retrospective study, antidepressant use at usual antidepressant doses was significantly and substantially associated with lower risk of intubation or death among adult patients hospitalized for COVID-19. 
Double-blind controlled randomized clinical trials of antidepressant medications for COVID-19 are needed.

\section{Data availability}

Data from the AP-HP Health Data Warehouse can be obtained with permission at https://eds.aphp.fr//.

Acknowledgements The authors thank the EDS APHP Covid consortium integrating the APHP Health Data Warehouse team as well as all the APHP staff and volunteers who contributed to the implementation of the EDS-Covid database and operating solutions for this database. Collaborators of the EDS APHP Covid consortium are: Pierre-Yves Ancel, Alain Bauchet, Nathanaël Beeker, Vincent Benoit, Mélodie Bernaux, Ali Bellamine, Romain Bey, Aurélie Bourmaud, Stéphane Breant, Anita Burgun, Fabrice Carrat, Charlotte Caucheteux, Julien Champ, Sylvie Cormont, Christel Daniel, Julien Dubiel, Catherine Ducloas, Loic Esteve, Marie Frank, Nicolas Garcelon, Alexandre Gramfort, Nicolas Griffon, Olivier Grisel, Martin Guilbaud, Claire Hassen-khodja, François Hemery, Martin Hilka, Anne Sophie Jannot, Jerome Lambert, Richard Layese, Judith Leblanc, Léo Lebouter, Guillaume Lemaitre, Damien Leprovost, Ivan Lerner, Kankoe Levi Sallah, Aurélien Maire, Mariefrance Mamzer, Patricia Martel, Arthur Mensch, Thomas Moreau, Antoine Neuraz, Nina Orlova, Nicolas Paris, Bastien Rance, Hélène Ravera, Antoine Rozes, Elisa Salamanca, Arnaud Sandrin, Patricia Serre, Xavier Tannier, Jean-marc Treluyer, Damien Van Gysel, Gaël Varoquaux, Jill Jen Vie, Maxime Wack, Perceval Wajsburt, Demian Wassermann, Eric Zapletal. We also thank Dr. Aude DELCUZE for her helpful comments on early versions of the article

Author contributions Study protocol: NH, MS-R, FL. Conceptualization: NH, MS-R, FL, NB, A-S J. Data curation: MS-R, RV, $\mathrm{NB}, \mathrm{A}-\mathrm{SJ}, \mathrm{AN}, \mathrm{NP}, \mathrm{CD}, \mathrm{AG}, \mathrm{GL}, \mathrm{MB}, \mathrm{AB}$. Formal analysis: NH, MS, RV. Methodology: NH, MS-R. Writing - original draft: NH. Writing review \& editing: NH, MS-R, FL, RV, NB, AN, CL, NP, CD, AG, GA, GL, MB, AB, ES, AB.

\section{Compliance with ethical standards}

Conflict of interest All authors have completed the Unified Competing Interest form (available on request from the corresponding author) and declare: no support from any organization for the submitted work; $\mathrm{NH}$ has received personal fees and non-financial support from Lundbeck, outside the submitted work. FL has received speaker and consulting fees from Janssen-Cilag, Euthérapie-Servier, and Lundbeck, outside the submitted work. CL reports personal fees and non-financial support from Janssen-Cilag, Lundbeck, Otsuka Pharmaceutical, and Boehringer Ingelheim, outside the submitted work. GA reports personal fees from Pfizer, Pierre Fabre and Lundbeck, outside the submitted work; other authors declare no financial relationships with any organization that might have an interest in the submitted work in the previous three years; no other relationships or activities that could appear to have influenced the submitted work.

Ethical approval This observational study using routinely collected data received approval from the Institutional Review Board of the AP-HP clinical data warehouse (decision CSE-20-20_COVID19, IRB00011591, April 8th, 2020). AP-HP clinical Data Warehouse initiative ensures patients' information and informed consent regarding the different approved studies through a transparency portal in accordance with European Regulation on data protection and authorization $n^{\circ} 1980120$ from National Commission for Information Technology and Civil Liberties (CNIL). All procedures related to this work adhered to the ethical standards of the relevant national and institutional committees on human experimentation and with the Helsinki Declaration of 1975, as revised in 2008.

Publisher's note Springer Nature remains neutral with regard to jurisdictional claims in published maps and institutional affiliations.

\section{References}

1. Hoertel N, Blachier M, Blanco C, Olfson M, Massetti M, Sánchez-Rico $\mathrm{M}$, et al. A stochastic agent-based model of the SARS-CoV-2 epidemic in France. Nat Med. 2020;26: $1417-21$

2. Hoertel N, Blachier M, Blanco C, Olfson M, Massetti M, Limosin F, et al. Facing the COVID-19 epidemic in NYC: a stochastic agent-based model of various intervention strategies. MedRxiv. 2020. 2020. https://doi.org/10.1101/2020.04.23.20076885.

3. Gordon DE, Jang GM, Bouhaddou M, Xu J, Obernier K, White $\mathrm{KM}$, et al. A SARS-CoV-2 protein interaction map reveals targets for drug repurposing. Nature. 2020;583:459-68.

4. Chevance A, Gourion D, Hoertel N, Llorca P-M, Thomas P, Bocher R, et al. Ensuring mental health care during the SARSCoV-2 epidemic in France: a narrative review. L'Encephale. 2020;46:S3-S13.

5. Stebbing J, Phelan A, Griffin I, Tucker C, Oechsle O, Smith D, et al. COVID-19: combining antiviral and anti-inflammatory treatments. Lancet Infect Dis. 2020;20:400-2.

6. Liu Y, Yan L-M, Wan L, Xiang T-X, Le A, Liu J-M, et al. Viral dynamics in mild and severe cases of COVID-19. The Lancet Infectious Diseases. 2020;20:656-7.

7. Hojyo S, Uchida M, Tanaka K, Hasebe R, Tanaka Y, Murakami $\mathrm{M}$, et al. How COVID-19 induces cytokine storm with high mortality. Inflamm Regen. 2020;40:1-7.

8. Köhler CA, Freitas TH, Stubbs B, Maes M, Solmi M, Veronese N, et al. Peripheral alterations in cytokine and chemokine levels after antidepressant drug treatment for major depressive disorder: systematic review and meta-analysis. Mol Neurobiol. 2018;55:4195-206.

9. Ye Q, Wang B, Mao J. The pathogenesis and treatment of theCytokine Storm'in COVID-19. J Infect. 2020;80:607-13.

10. Gulbins E, Palmada M, Reichel M, Lüth A, Böhmer C, Amato D, et al. Acid sphingomyelinase-ceramide system mediates effects of antidepressant drugs. Nat Med. 2013;19:934-8.

11. Carpinteiro A, Edwards MJ, Hoffmann M, Kochs G, Gripp B, Weigang S, et al. Pharmacological inhibition of acid sphingomyelinase prevents uptake of SARS-CoV-2 by epithelial cells. Cell Reports Med. 2020;1:100142.

12. Zimniak M, Kirschner L, Hilpert H, Seibel J, Bodem J. The serotonin reuptake inhibitor Fluoxetine inhibits SARS-CoV-2. BioRxiv. 2020. https://www.biorxiv.org/content/10.1101/2020. $06.14 .150490 \mathrm{v} 2$.

13. Lenze EJ, Mattar C, Zorumski CF, Stevens A, Schweiger J, Nicol $\mathrm{GE}$, et al. Fluvoxamine vs placebo and clinical deterioration in outpatients with symptomatic COVID-19: a randomized clinical trial. JAMA. 2020;324:2292-2300.

14. Cipriani A, Furukawa TA, Salanti G, Chaimani A, Atkinson LZ, Ogawa Y, et al. Comparative efficacy and acceptability of 21 antidepressant drugs for the acute treatment of adults with major depressive disorder: a systematic review and network metaanalysis. Focus. 2018;16:420-9.

15. Tham A, Jonsson U, Andersson G, Söderlund A, Allard P, Bertilsson G. Efficacy and tolerability of antidepressants in people aged 65 years or older with major depressive disorder-a systematic review and a meta-analysis. $J$ Affect Disord. 2016;205:1-12. 
16. Krause M, Gutsmiedl K, Bighelli I, Schneider-Thoma J, Chaimani A, Leucht S. Efficacy and tolerability of pharmacological and nonpharmacological interventions in older patients with major depressive disorder: A systematic review, pairwise and network meta-analysis. Eur Neuropsychopharmacol. 2019;29:1003-22.

17. Williamson E, Walker AJ, Bhaskaran KJ, Bacon S, Bates C, Morton CE, et al. OpenSAFELY: factors associated with COVID19-related hospital death in the linked electronic health records of 17 million adult NHS patients. MedRxiv. 2020. https://doi.org/10. 1101/2020.05.06.20092999.

18. Devlin J, Chang M-W, Lee K, Toutanova K. Bert: Pre-training of deep bidirectional transformers for language understanding. ArXiv. 2018. https://arxiv.org/abs/1810.04805.

19. Zhou F, Yu T, Du R, Fan G, Liu Y, Liu Z, et al. Clinical course and risk factors for mortality of adult inpatients with COVID-19 in Wuhan, China: a retrospective cohort study. Lancet. 2020;395:1054-62.

20. Ruan Q, Yang K, Wang W, Jiang L, Song J. Clinical predictors of mortality due to COVID-19 based on an analysis of data of 150 patients from Wuhan, China. Intensive Care Med. 2020;46:846-8.

21. Hur K, Price CP, Gray EL, Gulati RK, Maksimoski M, Racette $\mathrm{SD}$, et al. Factors associated with intubation and prolonged intubation in hospitalized patients with COVID-19. Otolaryngol Head Neck Surg. 2020;163:170-8.

22. Hoertel N, Sánchez-Rico M, Vernet R, Beeker N, Neuraz A, Alvarado JM, et al. Dexamethasone use and Mortality in Hospitalized Patients with Coronavirus Disease 2019: a Multicenter Retrospective Observational Study. MedRxiv. 2020. https://doi. org/10.1101/2020.10.23.20218172.

23. Haut Conseil de la Santé Publique. Statement on the management at home or in a care facility of suspected or confirmed Covid-19 patients. April 8, 2020. Statement on the management at home or in a care facility of suspected or confirmed Covid-19 patients. https://www.hcsp.frltextgreater.

24. Lagunas-Rangel FA. Neutrophil-to-lymphocyte ratio and lymphocyte-to-C-reactive protein ratio in patients with severe coronavirus disease 2019 (COVID-19): a meta-analysis. J Med Virol. 2020;92:1733-4.
25. Hoertel N, Sánchez-Rico M, Vernet R, Jannot A-S, Neuraz A, Blanco C, et al. Observational study of chlorpromazine in hospitalized patients with Covid-19. MedRxiv. 2020. 2020. https:// doi.org/10.1101/2020.07.15.20154310.

26. Hoertel N, Sánchez-Rico M, Vernet R, Jannot A-S, Neuraz A, Blanco C, et al. Observational study of haloperidol in hospitalized patients with Covid-19. MedRxiv. 2020. https://doi.org/10.1101/ 2020.07.15.20150490.

27. Robins JM, Hernán MÁ, Brumback B. Marginal structural models and causal inference in epidemiology. Epidemiology. 2000;11:550-60.

28. Geleris J, Sun Y, Platt J, Zucker J, Baldwin M, Hripcsak G, et al. Observational study of hydroxychloroquine in hospitalized patients with Covid-19. N. Engl J Med. 2020;382:2411-8.

29. Kaplan EL, Meier P. Nonparametric estimation from incomplete observations. J Am Stat Assoc. 1958;53:457-81.

30. Efron B. Nonparametric standard errors and confidence intervals. Can J Stat. 1981;9:139-58.

31. Hayasaka Y, Purgato M, Magni LR, Ogawa Y, Takeshima N, Cipriani A, et al. Dose equivalents of antidepressants: evidencebased recommendations from randomized controlled trials. J Affect Disord. 2015;180:179-84.

32. Von Elm E, Altman DG, Egger M, Pocock SJ, Gøtzsche PC, Vandenbroucke JP. The Strengthening the Reporting of Observational Studies in Epidemiology (STROBE) statement: guidelines for reporting observational studies. Ann Intern Med. 2007; 147:573-7.

33. Wang QQ, Kaelber DC, Xu R, Volkow ND. COVID-19 risk and outcomes in patients with substance use disorders: analyses from electronic health records in the United States. Mol Psychiatry. 2021;26:30-9.

34. Li L, Li F, Fortunati F, Krystal JH. Association of a prior psychiatric diagnosis with mortality among hospitalized patients with coronavirus disease 2019 (COVID-19) Infection. JAMA Netw Open. 2020;3:e2023282-e2023282.

35. Le Strat Y, Hoertel N. Correlation is no causation: gymnasium proliferation and the risk of obesity. Addiction. 2011;106:1871-2.

\section{Affiliations}

\section{Nicolas Hoertel $\oplus^{1,2,3} \cdot$ Marina Sánchez-Rico $\mathbb{D}^{1,4} \cdot$ Raphaël Vernet $\mathbb{D}^{5} \cdot$ Nathanaël Beeker ${ }^{6}$. Anne-Sophie Jannot ${ }^{3,5,7}$ - Antoine Neuraz ${ }^{7,8}$ - Elisa Salamanca ${ }^{9}$ - Nicolas Paris ${ }^{10,11}$. Christel Daniel $^{10,12}$. Alexandre Gramfort ${ }^{13} \cdot$ Guillaume Lemaitre $^{13} \cdot$ Mélodie Bernaux $^{14}$ - Ali Bellamine ${ }^{6}$. Cédric Lemogne $\mathbb{D}^{1,2,3}$. Guillaume Airagnes $^{1,2,3} \cdot$ Anita Burgun $^{7} \cdot$ Frédéric Limosin $^{1,2,3} \cdot$ On behalf of AP-HP / Universities / INSERM COVID- 19 Research Collaboration and AP-HP COVID CDR Initiative}

1 AP-HP.Centre-Université de Paris, Hôpital Corentin-Celton, Département Médico-Universitaire de Psychiatrie et Addictologie, 92130 Issy-les-Moulineaux, France

2 INSERM, Institut de Psychiatrie et Neurosciences de Paris, UMR_S1266, Paris, France

3 Université de Paris, Faculté de Santé, UFR de Médecine, Paris, France

4 Department of Psychobiology \& Behavioural Sciences Methods, Faculty of Psychology, Universidad Complutense de Madrid, Campus de Somosaguas, Pozuelo de Alarcon, Spain
5 AP-HP.Centre-Université de Paris, Hôpital Européen Georges Pompidou, Medical Informatics, Biostatistics and Public Health Department, F-75015 Paris, France

6 AP-HP.Centre-Université de Paris, Unité de Recherche clinique, Hôpital Cochin, Paris, France

7 INSERM, UMR_S1138, Cordeliers Research Center, Université de Paris, Paris, France

8 AP-HP.Centre-Université de Paris, Necker-Enfants Malades Hospital, Department of Medical Informatics, 75015 Paris, France

9 Banque Nationale de Données Maladies Rares, Campus Picpus, Département WIND (Web Innovation Données), Paris, France 
10 AP-HP, DSI-WIND (Web Innovation Données), Paris, France

11 LIMSI, CNRS, Université Paris-Sud and Université Paris-Saclay, F-91405 Orsay, France

12 Sorbonne University, University Paris 13, Sorbonne Paris Cité, INSERM UMR_S1142, F-75012 Paris, France
13 Université Paris-Saclay, INRIA, CEA, Palaiseau, France

14 Direction de la stratégie et de la transformation, AP-HP, Paris, France 